\title{
Machine learning model for diagnosing the stage of liver fibrosis in patients with chronic viral hepatitis $\mathrm{C}$
}

\author{
Valeriy V. Tsvetkov ${ }^{1}$, Ivan I. Tokin ${ }^{1,2}$, Dmitriy A. Lioznov ${ }^{1,3}$ \\ ${ }^{1}$ Smorodintsev Research Institute of Influenza, Saint Petersburg, Russia \\ ${ }^{2}$ North-Western State Medical University named after I.I. Mechnikov, Saint Petersburg, Russia \\ ${ }^{3}$ Pavlov First Saint Petersburg State Medical University, Saint Petersburg, Russia
}

\begin{abstract}
Aim. The purpose of the work was the development of a machine learning model for diagnosing the stage of liver fibrosis in patients with chronic viral hepatitis $\mathrm{C}$ according to the data of routine clinical examination.

Materials and methods. A total of 1240 patients with chronic viral hepatitis $\mathrm{C}$ was examined. A set of data obtained from 689 patients balancing by the stage of liver fibrosis was used for developing and testing machine learning models. 9 routine clinical parameters were selected as the most important predictors for determining the likelihood of liver fibrosis the 3-4 stages presence: age, height, weight and body mass index of the patient, the number of platelets in the clinical blood test, levels of alanine transaminase, aspartate transaminase, gamma-glutamyltransferase, and total bilirubin in a biochemical blood test.

Results. The accuracy of the developed method for determining the 3-4 stages of liver fibrosis in patients with chronic viral hepatitis $\mathrm{C}$ in comparison with the «gold standard» of diagnosis (liver biopsy) was $80.56 \%$ (95\% CI: $69.53-88.94 \%$ ), sensitivity - 66.67\%, specificity $-94.44 \%$.

Conclusion. The developed method is an alternative to more expensive and geographically inaccessible studies. The method does not require the purchase of additional equipment or software, as well as additional laboratory tests, when used in real clinical practice. The introduction of the method into clinical practice can help to solve the problem of low material and territorial availability of diagnostic tests and allow determining the stage of liver fibrosis in patients with chronic viral hepatitis C.
\end{abstract}

Keywords: Hepatitis C virus, Chronic viral hepatitis C, Liver fibrosis, Liver cirrhosis, Diagnosis of liver fibrosis, Machine learning

\section{Introduction}

There are 71 million people in the world infected with the hepatitis C virus (HCV), which is approximately $1 \%$ of the world's population [1]. At present, it is known that chronic infection develops in $60-85 \%$ of cases after acute viral hepatitis C [2]. Patients with chronic viral hepatitis $\mathrm{C}(\mathrm{CHC})$ are at the risk of developing life-threatening conditions such as liver cirrhosis and hepatocellular carcinoma [1,3]. Currently, there is increasing world mortality associated with $\mathrm{CHC}$ because of the growth of the population of longterm ill patients with severe liver fibrosis [4]. 
According to the experts from the World Health Organization (WHO), in 2016 approximately 399 thousand people died from hepatitis $\mathrm{C}$ in the world, mainly as a result of the development of liver cirrhosis and hepatocellular carcinoma [1].

Liver damage develops in the absolute majority of the patients with $\mathrm{CHC}$ and is an independent risk of premature death of the patients (RR 12.48; 95\% CI 9.34-16.66) [5]. Liver cirrhosis and hepatocellular carcinoma are the leading causes of CHC patient's deaths [1, 2]. In this regard, in the absolute majority of cases, the stage of liver fibrosis is the main indicator of the progression of this disease, which determines the further tactics of patient management and the priorities of therapy [6]. Thus, the material and territorial availability of methods for diagnosing the stage of liver fibrosis is a prerequisite for improving the quality of medical care for the patients with $\mathrm{CHC}$, as well as reducing the mortality and mortality rate associated with this disease.

The «gold standard» for determining the stage of liver fibrosis of the patients with CHC is liver biopsy. However, due to its inherent drawbacks, less invasive and faster methods such as transient elastography (Fibroscan $\mathrm{R}$ ), B-mode ultrasound and Fibrotest $\mathrm{R}$ ) are mostly commonly used in real clinical practice. In most cases, such methods allow determining the stage of liver fibrosis and refuse to perform a liver biopsy, but the accuracy of such methods is lower. The sensitivity of transient elastography for determining the 3-4 stages of liver fibrosis in patients with $\mathrm{CHC}$ is from 68 to $89 \%$ (average - 80.8\%), specificity - from 85 to $93 \%$ (average - 89.6\%) [7]. The sensitivity of point and two-dimensional shear wave elastography (2D-SWE) is from 84 to $97 \%$ (average - 90.6\%), specificity - from 89 to $95 \%$ (average - 92.7\%) [7]. In addition, Fibroscan(R, Fibrotest(R, Hepascore@ and Fibrometer@ are commercial patented medical technologies, while Fibroscan $\mathrm{R}$, B-mode ultrasound and magnetic resonance elastography require the purchase of expensive equipment, which leads to a high cost of diagnostics and its low material and territorial availability.

More accessible diagnostic methods are calculated indices APRI, FIB-4, MDA, FORNS and others, based on the determination of indirect routine clinical and laboratory markers. Such indices are calculated using simple mathematical formulas developed with the help of algorithms for classical statistical data analysis. The APRI and FIB-4 indices are recommended by the experts of the European Professional Association for the Study of Liver Diseases (EASL) to determine the 4 stage of liver fibrosis of the patients with $\mathrm{CHC}$ in routine clinical practice [6, 8]. Meanwhile, in terms of their accuracy, sensitivity and specificity such methods are inferior to invasive and instrumental analogues [9-16].

Mathematical models developed with modern machine learning algorithms are generally more efficient than simple computed indices. The material and territorial availability of the methods based on the use of machine learning models, as in cases of simple calculated indices, can be achieved through application of a limited set of indirect routine clinical and laboratory 
markers. In its turn, increasing the information content of such methods in relation to the indicators of the information content of the APRI and FIB-4 tests can be achieved by using modern machine learning algorithms, analyzing and taking into account complex relationships between all predictors used.

The aim of this study was to develop a machine learning model for determining the 3-4 stage of liver fibrosis of the patients with $\mathrm{CHC}$ according to the data of routine clinical examination.

\section{Materials and methods}

An observational retrospective study was carried out in 2020 by the staff of the Smorodintsev Research Institute of Influenza. The research protocol was approved by the local ethics committee of the Smorodintsev Research Institute of Influenza on February 25, 2020. The primary medical documentation of the study was the patient's observation cards at the Leningrad Region Center for Prevention and Control of AIDS and Communicable Diseases. All medical procedures were performed prior to the study as part of the routine clinical practice of outpatient management of the patients with CHC.

At the first stage of the study, a database was formed containing depersonalized anamnestic and clinical and laboratory data on 1240 patients with $\mathrm{CHC}$, of which males $-55.32 \%$ ( $\mathrm{n}=$ $686)$, females $-44.68 \%(\mathrm{n}=554)$ was formed. The median age of the patients was 42.00 years $(33.00-51.00$ years $)$, the average body mass index was $26.03 \mathrm{~kg} / \mathrm{m}^{2}\left(23.24-29.22 \mathrm{~kg} / \mathrm{m}^{2}\right)$. The diagnosis of «Chronic viral hepatitis C» in all the patients was first established at least 6 months before the date of examination on the basis of positive results of blood tests for total antibodies to HCV proteins and HCV RNA. None of the patients had severe comorbidities in the stage of decompensation, as well as co-infections caused by hepatitis A virus, hepatitis $B$ virus and human immunodeficiency virus. The database did not include information on patients with chronic liver diseases of other etiology, such as cytomegalovirus and Epstein - Barr viral infections, hemochromatosis, autoimmune hepatitis, alcoholic and non-alcoholic fatty liver disease, biliary tract disease and toxic hepatitis. Also, among the patients whose information was entered into the database, there were no patients with hepatocellular carcinoma and those who abused alcohol, injecting drugs or narcotic drugs taken by inhalation during the last 6 months.

The following information was entered into the study database: gender, age, height and weight of the patient, levels of platelets, hemoglobin, erythrocytes, leukocytes, absolute content of neutrophils and lymphocytes in a clinical blood test, erythrocyte sedimentation rate (ESR), levels of alanine transaminase (ALT), aspartate transaminase (AST), gamma-glutamyltransferase (GGT), total bilirubin, total protein, albumin, creatinine, amylase, triglycerides, cholesterol, alkaline phosphatase (ALP), iron and glucose in a biochemical blood test, as well as prothrombin index (PTI), genotype HCV, viral load and liver fibrosis stage on the Metavir scale. 


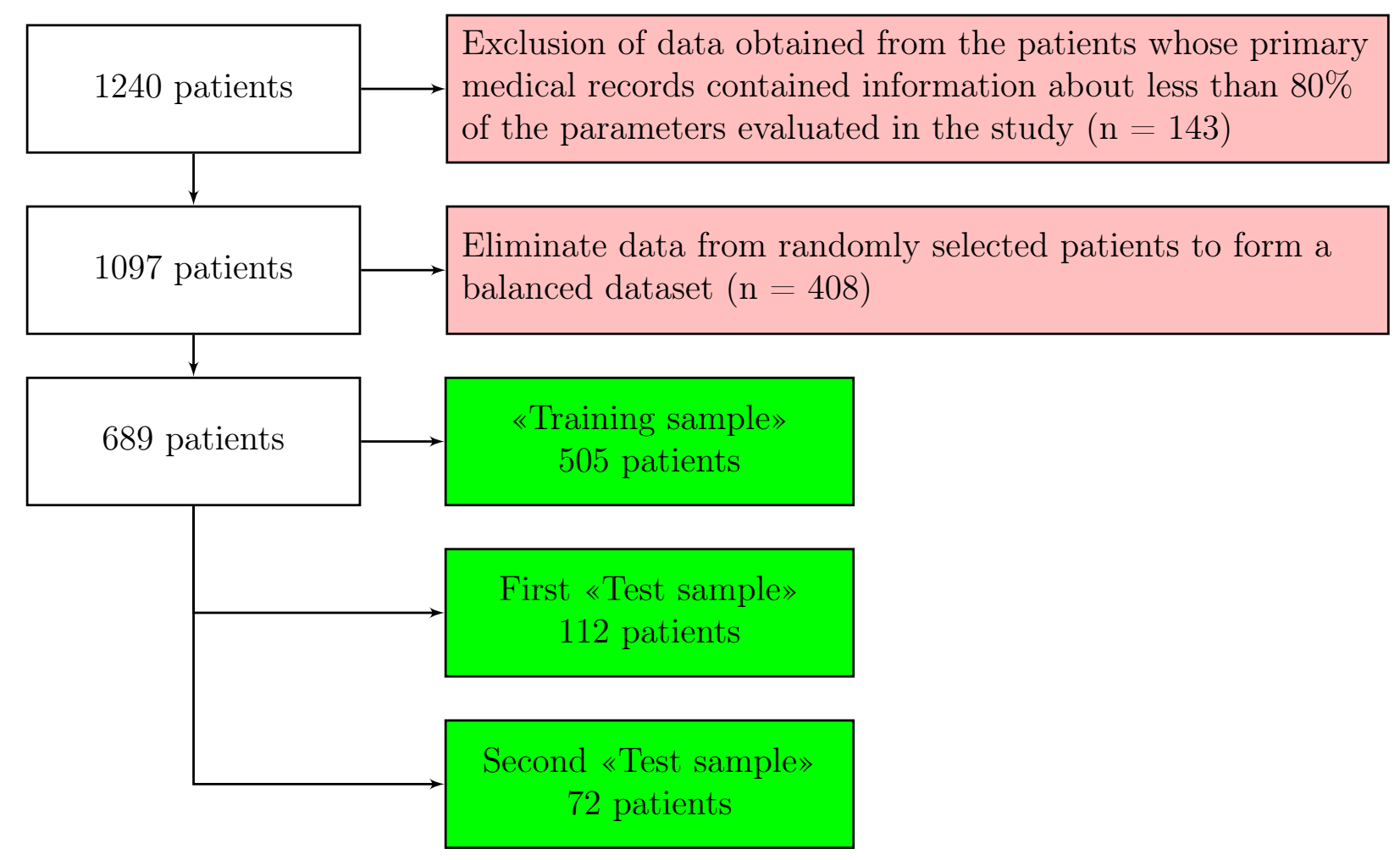

Figure 1. Data selection rules

The collected information was checked for filling errors and typos, as well as data standardization, checking for normality of distribution and homogeneity of variance of quantitative values at the stage of data analysis and processing. To form a stage-balanced sample of liver fibrosis, data on 551 patients were excluded from the initial data set. The remaining 689 patients were randomly divided into three groups in a ratio of 4: 1: 1 (Figure 1). Traditionally, the terms «training sample» and «test sample» are used to name groups / data samples used in the development of machine learning models. The «training sample» data are used to develop and validate a machine learning model. In turn, the data of the «test sample» are not used directly in the process of training the model, but they allow to test and control the model.

Randomly, taking into account the stratification by stage of liver fibrosis, the data were obtained from 505 patients with $\mathrm{CHC}$ and selected into the «training sample», of which males $55.25 \%(\mathrm{n}=279)$, females $-44.75 \%(\mathrm{n}=226)$. The median age of the patients was 45.00 years (36.00-54.00 years), the average body mass index $-26.67 \mathrm{~kg} / \mathrm{m}^{2}\left(23.46-29.39 \mathrm{~kg} / \mathrm{m}^{2}\right)$. Genotype 1 was established in $56.10 \%(\mathrm{n}=271)$ patients, genotype $3-$ in $37.47 \%(\mathrm{n}=181)$ patients. Liver fibrosis of the 0-2 stages was detected in $49.90 \%(\mathrm{n}=252)$ patients, liver fibrosis of the 3-4 stages - in $50.10 \%(\mathrm{n}=253)$ patients of the «training sample» (Table 1$)$. The stage of liver fibrosis in all patients of the «training sample» was determined by the method of liver transient elastography using the FibroScan R 402 device. 
Table 1. Comparative characteristics of patients in three groups

\begin{tabular}{lccc}
\hline Indicators & $\begin{array}{c}\text { Training } \\
\text { sample } \\
(\mathrm{n}=505)\end{array}$ & $\begin{array}{c}\text { First Test } \\
\text { sample } \\
(\mathrm{n}=112)\end{array}$ & $\begin{array}{c}\text { Second Test } \\
\text { sample } \\
(\mathrm{n}=72)\end{array}$ \\
\hline Sex & & & \\
\hline Male & $279 / 55.25$ & $69 / 61.61$ & $37 / 51.39$ \\
Female & $226 / 44.75$ & $43 / 38.39$ & $35 / 48.61$ \\
Age, years & $45.00(36.00-54.00)$ & $43.00(33.75-52.25)$ & $35.50(29.75-47.25)$ \\
Height, cm & $170.50(164.00-178.00)$ & $172.00(166.80-180.00)$ & $171.00(163.00-178.00)$ \\
Weight, $\mathrm{kg}$ & $78.00(67.00-90.00)$ & $78.50(68.75-90.00)$ & $72.00(63.00-73.00)$ \\
BMI, $\mathrm{kg} / \mathrm{m}^{2}$ & $26.67(23.46-29.39)$ & $26.48(23.66-29.67)$ & $23.95(21.78-27.76)$
\end{tabular}

HCV genotype

\begin{tabular}{lccc}
\hline 1 genotype & $271 / 56.10$ & $49 / 47.57$ & $41 / 60.30$ \\
2 genotype & $31 / 6.42$ & $3 / 2.91$ & $4 / 5.88$ \\
3 genotype & $181 / 37.47$ & $51 / 49.52$ & $23 / 33.82$
\end{tabular}

Stage of liver fibrosis

\begin{tabular}{lccc}
\hline 0 stage & $84 / 16.63$ & $19 / 16.96$ & $12 / 16.66$ \\
1 stage & $86 / 17.03$ & $19 / 16.96$ & $12 / 16.67$ \\
2 stage & $82 / 16.24$ & $18 / 16.07$ & $12 / 16.67$ \\
3 stage & $122 / 24.16$ & $27 / 24.11$ & $20 / 27.78$ \\
4 stage & $131 / 25.94$ & $29 / 25.89$ & $16 / 22.22$ \\
Laboratory parameters & & \\
\hline Platelets, $10^{9} / 1$ & $187.14(141.73-235.11)$ & $183.00(133.40-221.00)$ & $181.00(141.80-228.80)$ \\
ALT, U/l & $76.0(45.00-141.00)$ & $67.96(48.86-109.28)$ & $79.50(54.18-117.25)$ \\
AST, U/l & $59.40(37.41-105.42)$ & $57.01(38.98-92.02)$ & $56.81(43.00-79.52)$ \\
GGT, U/l & $49.67(28.81-88.85)$ & $49.17(31.32-83.03)$ & $47.00(31.00-99.70)$ \\
Tot. bil., mmol/1 & $14.24(10.60-19.86)$ & $13.00(9.97-19.59)$ & $15.75(12.76-20.79)$ \\
\hline
\end{tabular}

Note: in the table, group quantitative indicators are given as a median indicating the interquartile range $-\mathrm{Me}$ (LQ - HQ), qualitative (categorical) - in the form of abs. / \%.

In the first «test sample», randomly, taking into account stratification by the stage of liver fibrosis, information about 112 patients was selected, where: males $-61.61 \%(\mathrm{n}=69)$, females - 38.39\% $(\mathrm{n}=43)$. The average age of the patients in the first «test sample» was 43.00 years 
(33.75-52.25 years), the average body mass index $-26.48 \mathrm{~kg} / \mathrm{m}^{2}\left(23.66-29.67 \mathrm{~kg} / \mathrm{m}^{2}\right)$. Liver fibrosis of the 0-2 stages was detected in $50.00 \%(\mathrm{n}=56)$ patients, liver fibrosis of the $3-4$ stages was found in $50.00 \%(\mathrm{n}=56)$ patients. The stage of liver fibrosis in all patients of the first «test sample» was determined by the method of transient liver elastography using the FibroScan R 402 device.

In the second «test sample», randomly, taking into account the stratification by the stage of liver fibrosis, the data obtained from 72 patients were selected, where: males $-51.39 \%$ ( $\mathrm{n}=$ $37)$, females - 48.61\% ( $\mathrm{n}=35)$. The average age of the patients of the second «test sample» was 35.50 years $(29.75-47.25$ years $)$, the average body mass index $-23.95 \mathrm{~kg} / \mathrm{m}^{2}(21.78$ $\left.27.76 \mathrm{~kg} / \mathrm{m}^{2}\right)$. Hepatic fibrosis of the $0-2$ stages was detected in $50.00 \%(\mathrm{n}=36)$ patients, liver fibrosis of the $3-4$ stages was found in $50.00 \%(\mathrm{n}=36)$ patients. The stage of liver fibrosis in all patients of the second «test sample» was determined using the «gold standard» for diagnosing liver biopsy.

Only the data of the «training sample» were used with 5-fold crossvalidation at the stage of developing machine learning models for the selection of predictors, optimization of hyperparameters and training models. For the development of preliminary machine learning models, all clinical indicators entered into the database were used as predictors, and the determined outcomes were the presence or absence of the 3-4 stages liver fibrosis in the patient according to the Metavir scale. The relative influence of each predictor on the test accuracy was estimated to select the optimal set of predictors. The predictors with the largest mean loss in loss function score were considered as most important. To develop the final machine learning model, the data containing information on the level of ALT, AST, GGT and total bilirubin in the biochemical blood test were preliminarily logarithm, after which all initial data were normalized in the range from 0 to 1 .

At the stage of testing machine learning models, the informativeness indicators of methods based on the use of preliminary and final machine learning models, as well as methods based on the calculation of APRI and FIB-4 indices, were determined, which made it possible to conduct a comparative assessment. The data of the two «test samples» were used only once to determine the indicators of the information content of the test.

Mathematical and statistical processing of the obtained data was carried out using the software environment for statistical analysis «R». To balance the data and split it into groups, the «createDataPartition» function from the «caret» package was used. The median values were calculated as measures of the central trend. Quartile values were calculated as measures of data variability. The normality of the distribution of quantitative traits was assessed using the Shapiro - Wilk test. The homogeneity of variances of quantitative traits was assessed using the Bartlett test. To assess the differences between the two samples in terms of qualitative (categorical) features, contingency tables were formed and Fisher's exact test was used. The 
nonparametric Mann - Whitney test was used to assess the differences between two samples for quantitative characteristics. Indicators of the method informativeness, such as accuracy with the definition of 95\% confidence interval, sensitivity and specificity, Cohen's Kappa coefficient, predictive value of positive and negative test results, as well as the area under the ROC curve, were calculated using the corresponding functions from the packages «caret» and «ROCR». Gradient boosting machine learning models were developed in the «R» statistical analysis software using the classic «gbm» package. The exponential function AdaBoost was used as the loss function. The «scale» function was used to normalize the data in the range from 0 to 1.

\section{Results}

Using machine learning algorithms, we have developed several models to determine the likelihood of the presence of the 3-4 stages of liver fibrosis in patients with CHC (Table 2).

Table 2. Indicators of informativeness of methods based on the use of preliminary and final machine learning models

\begin{tabular}{lcccccccc}
\hline \multirow{2}{*}{ Methods } & F3-4 & F0-2 & Kappa & Sens & Spec & PPV & NPV & Acc \\
& $($ abs. $)$ & $($ abs. $)$ & & $(\%)$ & $(\%)$ & $(\%)$ & $(\%)$ & $(\%)$ \\
\hline
\end{tabular}

On the data of the «training sample»

$\begin{array}{ccccccccc}\text { Model } 1 & & & & & & & & \\ \geq 0.5 & 227 & 19 & 0.8218 & 89.72 & 92.46 & 92.28 & 89.96 & 91.09 \\ <0.5 & 26 & 233 & & & & & & \\ \text { Model } 2 & & & & & & & & \\ \geq 0.5 & 211 & 23 & & & & & \\ <0.5 & 42 & 229 & 0.7426 & 83.40 & 90.87 & 90.17 & 84.50 & 87.13\end{array}$

On the data of the first «test sample» 1

\begin{tabular}{ccccccccc}
\hline $\begin{array}{c}\text { Model } 1 \\
\geq 0.5\end{array}$ & 42 & 8 & & & & & & \\
$\quad$ & & & & & & & \\
$<0.5$ & 14 & 48 & 0.6071 & 75.00 & 85.71 & 84.00 & 77.42 & 80.36 \\
Model 2 & & & & & & & & \\
$\geq 0.5$ & 42 & 5 & 0.6607 & 75.00 & 91.07 & 89.36 & 78.46 & 83.04 \\
$<0.5$ & 14 & 51 & & & & & & \\
\hline
\end{tabular}

Note: Model 1 - preliminary model; Model 2 - the final model; Kappa - Cohen's Kappa coefficient; Sens — sensitivity; Spec - specificity; PPV - positive predictive value; NPV - negative predictive value; Acc accuracy. 
The accuracy of the method based on the use of a preliminary machine learning model (model 1) on the data of the «training sample» was $91.09 \%$ (95\% CI: 88.26-93.43\%), on the data of the first «test sample» - 80.36\% (95\% CI: 71.78-87.26\%).

Figure 2 shows the levels of the relative influence of all predictors on the average decrease in the loss function. It has been established that the most important predictors necessary for the most accurate determination of the probability of the presence of the 3-4 stages of liver fibrosis in patients with $\mathrm{CHC}$ are the number of platelets in the clinical blood test, the levels of AST and GGT in the biochemical blood test, as well as the patient's age.

The exclusion from the set of predictors of clinical indicators that did not or weakly affected the accuracy of the forecast, as well as indicators, the determination of which required significant additional economic costs, made it possible to develop the final machine learning model (model 2), taking into account only 9 of 28 parameters: age, the patient's height, weight and BMI, the number of platelets in the clinical blood test, the levels of ALT, AST, GGT and total bilirubin in the biochemical blood test.

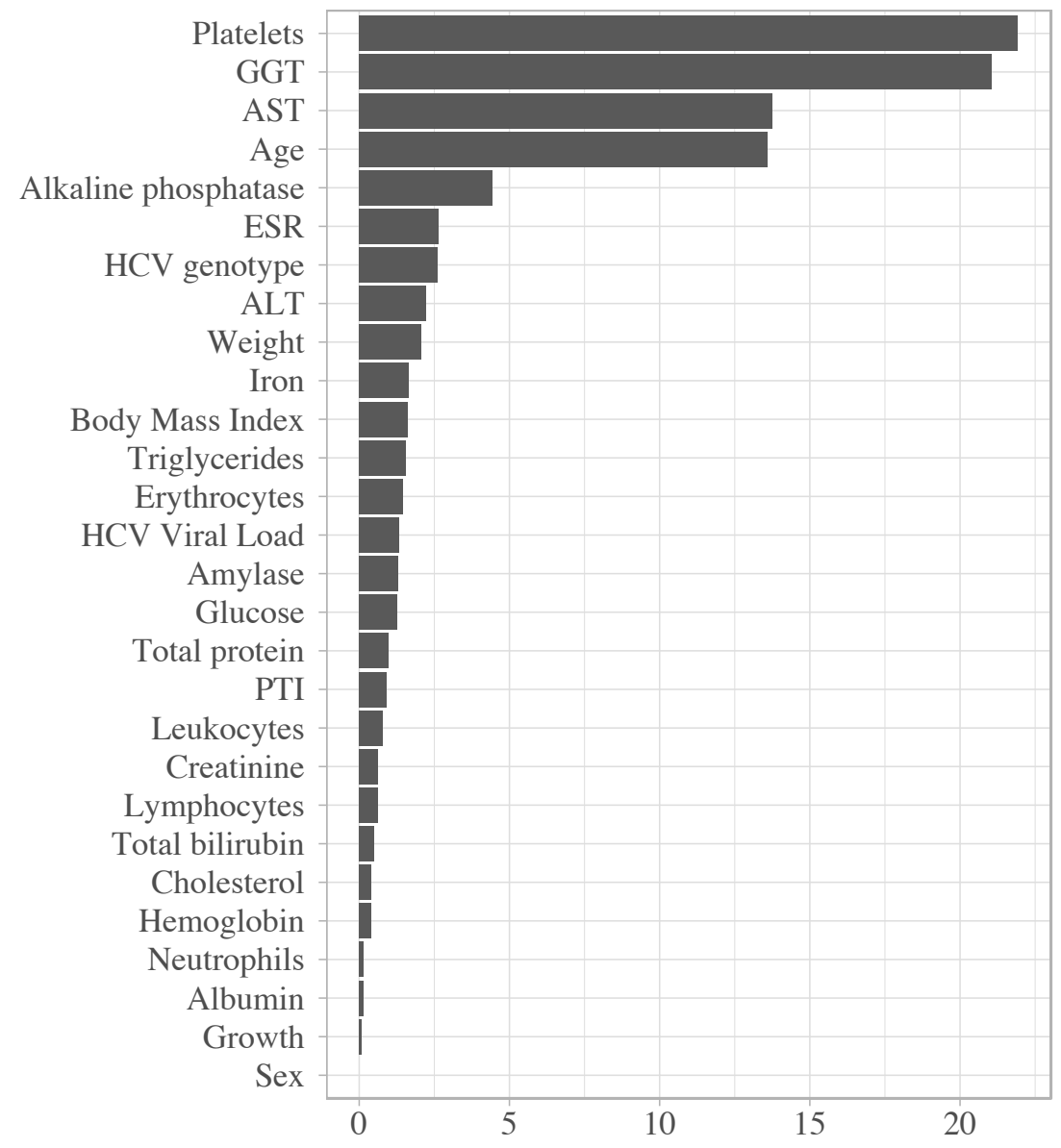

Figure 2. Relative importance of predictors 
Indicators of the methods informativeness based on the use of the final machine learning model (model 2) and the calculation of the APRI and FIB-4 indices on the data of the first «test sample» are presented in Table 3. The accuracy of the method based on model 2 in comparison with transient liver elastography was $83.04 \%$ (95\% CI: 74.78-89.47\%), sensitivity - $75.00 \%$, specificity - 91.07\%. Compared to methods based on the calculation of APRI and FIB-4 indices, the method based on the final machine learning model was characterized by higher accuracy and area under the ROC curve, with more optimal ratio of sensitivity and specificity.

Table 3. Indicators of the methods informativeness for determining the 3-4 stages of liver fibrosis in patients with $\mathrm{CHC}$ in comparison with transient liver elastography

\begin{tabular}{lcccc}
\hline Methods & Acc $(\%)$ & Sens $(\%)$ & Spec (\%) & AUC \\
\hline Model 2 (cut-off value $=0.50)$ & 83.04 & 75.00 & 91.07 & 0.91 \\
APRI (cut-off value $=1.00)$ & 78.38 & 83.93 & 72.73 & 0.87 \\
FIB-4 (cut-off value $=3.25)$ & 74.77 & 49.09 & 100.00 & 0.88 \\
\hline
\end{tabular}

Note: Model 2 - final model; Acc - accuracy; Sens - sensitivity; Spec - specificity; AUC - area under the ROC curve.

Indicators of the methods informativeness based on the use of the final machine learning model (model 2) and the calculation of APRI and FIB-4 indices on the data of the second «test sample» are presented in Table 4. The accuracy of the method based on model 2 in comparison with the «gold standard» for diagnosing the stage of liver fibrosis (liver biopsy) was 80.56\% (95\% CI: $69.53-88.94 \%$ ), sensitivity - 66,67\%, specificity - 94.44\%. The methods informativeness indicators based on the calculation of the APRI and FIB-4 indices were significantly less $70.83 \%$ and $59.72 \%$, respectively.

Table 4. Indicators of the methods informativeness for determining the 3-4 stages of liver fibrosis in patients with $\mathrm{CHC}$ in comparison with liver biopsy

\begin{tabular}{lcccc}
\hline Methods & Acc $(\%)$ & Sens $(\%)$ & Spec (\%) & AUC \\
\hline Model 2 (cut-off value $=0.50)$ & 80.56 & 66.67 & 94.44 & 0.87 \\
APRI (cut-off value $=1.00)$ & 70.83 & 86.11 & 55.56 & 0.82 \\
FIB-4 (cut-off value $=3.25)$ & 59.72 & 19.44 & 100.00 & 0.81 \\
\hline
\end{tabular}

Note: Model 2 - final model; Acc - accuracy; Sens - sensitivity; Spec - specificity; AUC - area under the ROC curve. 


\section{Discussion}

The stage of liver fibrosis is a reliable indicator of the degree of liver damage and the progression of $\mathrm{CHC}$, which determines the further tactics of patient management. The stage of liver fibrosis is one of the most important indicators determining the sequence of antiviral therapy and the choice of an antiretroviral therapy regimen in patients with coinfection with $\mathrm{CHC}$ and HIV infection. The stage of liver fibrosis can also determine the tactics of patient management and the choice of treatment regimens in patients with combined pathology of CHC and tuberculosis, because of the presence of hepatotoxicity in many antituberculosis drugs. Meanwhile, the material and territorial availability of methods for diagnosing the stage of liver fibrosis in patients with CHC remains low, especially in remote regions. In this regard, scientific and practical activities aimed at developing and introducing new effective methods for diagnosing the stage of liver fibrosis, which do not require the purchase of expensive equipment, additional sets of reagents and other consumables into clinical practice, seem to us extremely relevant.

The «FP-Test» method developed by us is based on the use of a mathematical model of machine learning, which determines the probability of a patient having a presence of the 3-4 stages of liver fibrosis according to the Metavir scale. The initial data necessary for calculating the probability are the results of a routine clinical examination: age, height and weight of the patient, the number of platelets in the clinical blood test, the levels of ALT, AST, GGT and total bilirubin in the biochemical blood test. A computer interface has been developed for entering clinical and laboratory parameters and obtaining research results for the convenience of using the method. The interface for informational use is presented on the official website: http://ai.influenza.spb.ru/en. The accuracy of the method based on the use of the machine learning model developed by us to determine the 3-4 stages of liver fibrosis on the Metavir scale in patients with CHC in comparison with the «gold standard» of diagnosis (liver biopsy) is $80.56 \%$ (95\% CI: $69.53-88.94 \%$ ), sensitivity - 66.67\%, specificity - $94.44 \%$.

The new method does not require the purchase of additional equipment or software, as well as additional laboratory tests. Unlike simple calculated indices, the work of the method is based on the use of a complex mathematical model developed using modern machine learning algorithms, which makes it possible to achieve higher and balanced indicators of the information content of the test. The introduction of the method into clinical practice can help solve the problem of low material and territorial availability of diagnostic tests that allow determining the stage of liver fibrosis in patients with $\mathrm{CHC}$.

Despite the fact that our study did not use unrepresentative and unbalanced samples, did not unreasonably exclude data, and used two independent test samples to estimate the accuracy, the developed machine learning model requires external verification. It also seems appropriate to conduct further research to assess the clinical and economic efficiency of the method. 


\section{Declarations}

Author Contributions: Conceptualization, all authors; Methodology, Tsvetkov V.V., Tokin I.I.; Software, Tsvetkov V.V., Tokin I.I.; Validation, Tsvetkov V.V., Tokin I.I.; Formal Analysis, Tsvetkov V.V., Tokin I.I.; Investigation, all authors; Resources, all authors; Data Curation, Tsvetkov V.V.; Writing - Original Draft Preparation, Tsvetkov V.V., Tokin I.I.; Writing - Review \& Editing, Tsvetkov V.V.; Visualization, Tsvetkov V.V.; Supervision, Tokin I.I., Liosnov D.A.; Project Administration, Tokin I.I.

Funding: The study was carried out as part of a research work on a state order (НИОКТР AAAA-A18-118022790087-7).

Data sharing: The datasets analyzed during this study are available from the authors on reasonable request.

Conflicts of Interest: All authors declared no conflict of interest.

\section{References}

1. Global hepatitis report 2017. World Health Organization, 2017.

2. Westbrook RH and Dusheiko G. Natural history of hepatitis C. Journal of hepatology 2014;61:58-68.

3. Chen SL and Morgan TR. The natural history of hepatitis C virus (HCV) infection. International journal of medical sciences 2006;3:47.

4. Deuffic-Burban S, Poynard T, Sulkowski M, and Wong J. Estimating the future health burden of chronic hepatitis $\mathrm{C}$ and human immunodeficiency virus infections in the United States. Journal of viral hepatitis 2007;14:107-15.

5. Lee $\mathrm{MH}$ et al. Chronic hepatitis $\mathrm{C}$ virus infection increases mortality from hepatic and extrahepatic diseases: a community-based long-term prospective study. The Journal of infectious diseases 2012;206:469-77.

6. The Study of The Liver EA for et al. EASL recommendations on treatment of hepatitis C 2018. Journal of hepatology 2018;69:461-511.

7. Kennedy P et al. Quantitative elastography methods in liver disease: current evidence and future directions. Radiology 2018;286:738-63.

8. Study of the Liver EA for the et al. EASL-ALEH Clinical Practice Guidelines: Noninvasive tests for evaluation of liver disease severity and prognosis. Journal of hepatology 2015;63:237-64.

9. Castéra L et al. Prospective comparison of transient elastography, Fibrotest, APRI, and liver biopsy for the assessment of fibrosis in chronic hepatitis C. Gastroenterology 2005;128:34350 . 
10. Férard $\mathrm{G}$ et al. Intermethod calibration of alanine aminotransferase (ALT) and $\gamma$-glutamyltransfera (GGT) results: application to Fibrotest@ and Actitest@ scores. Clinical Chemistry and Laboratory Medicine (CCLM) 2006;44:400-6.

11. Leroy V et al. Diagnostic accuracy, reproducibility and robustness of fibrosis blood tests in chronic hepatitis C: a meta-analysis with individual data. Clinical biochemistry 2008;41:136876.

12. Poynard $\mathrm{T}$ et al. Biochemical markers of liver fibrosis in patients infected by hepatitis $\mathrm{C}$ virus: longitudinal validation in a randomized trial. Journal of Viral Hepatitis 2002;9:12833.

13. Shaheen AAM and Myers RP. Diagnostic accuracy of the aspartate aminotransferase-toplatelet ratio index for the prediction of hepatitis C-related fibrosis: A systematic review. Hepatology 2007;46:912-21.

14. Lin $\mathrm{ZH}$ et al. Performance of the aspartate aminotransferase-to-platelet ratio index for the staging of hepatitis C-related fibrosis: an updated meta-analysis. Hepatology 2011;53:72636 .

15. Poynard $\mathrm{T}$ et al. Biomarkers of liver injury for hepatitis clinical trials: a meta-analysis of longitudinal studies. Antiviral therapy 2010;15:617.

16. Duarte-Rojo A, Altamirano JT, and Feld JJ. Noninvasive markers of fibrosis: key concepts for improving accuracy in daily clinical practice. Annals of Hepatology 2012;11:426-39. 